\title{
Bedside emergency department ultrasonography availability and use for blunt abdominal trauma in Canadian pediatric centres
}

\author{
Robin Cardamore, MDCM; Joe Nemeth, MDCM; Christine Meyers, MDCM
}

\section{ABSTRACT}

Objectives: To quantify the current availability and use of bedside emergency department ultrasonography (EDUS) for blunt trauma at Canadian pediatric centres and to identify any perceived barriers to the use of bedside EDUS in such centres.

Methods: An electronic survey was sent to 162 pediatric emergency physicians and 12 site directors from the 12 pediatric emergency departments across Canada.

Results: Ninety-two percent (11 of 12) of centres completed the survey. The individual physician response rate was $65 \%$ (106 of 162), with $100 \%$ of site directors responding. Ultrasound machines were available in $45 \%$ (5 of 11) of centres. Forty-two percent (32 of 77) of emergency physicians working in equipped pediatric centres used bedside EDUS to evaluate blunt abdominal trauma (BAT). In the subgroup of staff who also worked at adults sites, the frequency of ultrasonography use for the evaluation of pediatric BAT was $75 \%$. In the $55 \%$ (6 of 11) of centres without ultrasonography, $88 \%$ of staff intend to incorporate its use in the future and $81 \%$ indicated that they believed the incorporation of ultrasonography would have a positive impact on patient care. The main perceived barriers to the use of ultrasonography in the evaluation of BAT were a lack of training $(41 \%)$ and a lack of equipment $(26 \%)$.

Conclusion: Bedside EDUS is currently used in almost half of pediatric trauma centres, a frequency that is significantly lower than adult centres. Physicians in pediatric centres who use ultrasonography report that it has a high utility, and a great majority of physicians at pediatric centres without EDUS plan to incorporate it in the future. The main reported barriers to its use are a lack of training and a lack of equipment availability.

Keywords: abdominal, pediatric, prevalence, trauma, ultrasonography
Blunt abdominal injuries are a frequent reason for presentation of children to the emergency department (ED), ${ }^{1}$ and blunt trauma is the most common cause of death and disability in children greater than 1 year of age. ${ }^{1}$ The evaluation of injured children with suspected blunt abdominal trauma (BAT) is challenging as historical, physical, and laboratory findings are of limited utility, thus often necessitating further testing.

Abdominal computed tomography (CT) is currently the modality of choice for the definitive evaluation of stable pediatric patients with suspected intra-abdominal injuries (IAIs). However, CT requires that patients be cooperative and transportable, which is often problematic in the pediatric setting. Given the high rate of negative scans $^{2,3}$ and the association of low-dose radiation exposure in childhood leading to an increase in the lifetime risk for fatal cancers, ${ }^{4-7}$ the need for a safer and easier imaging modality is evident.

Bedside emergency department ultrasonography (EDUS) offers the benefits of being portable, quick, noninvasive, repeatable, and without risk of radiation. EDUS has been shown to be useful in adults as a screening modality for suspected IAI, ${ }^{8-10}$ with a recent randomized controlled trial showing a $64 \%$ reduction in time to operative care compared to CT alone. ${ }^{11}$ The applicability of EDUS in children is less well established. Two recent meta-analyses concluded that pediatric EDUS can adequately identify IAI, with positive likelihood ratios of 22.9 (95\% CI 17.2-30.5) and 22.0 (95\% CI 15.1-32.1), ${ }^{12,13}$ but that negative scans are insufficient to rule out injuries, with negative

From the Emergency Medicine Residency Training Program, McGill University, Montreal, OC.

Correspondence to: Dr. Robin Cardamore, McGill University Emergency Medicine Residency Training Program, Room A4.62, Royal Victoria Hospital, 687 Pine Avenue West, Montreal, QC H3A 1A1; robin.cardamore@mail.mcgill.ca.

Submitted September 17, 2010; Revised January 11, 2011, and January 19, 2011; Accepted January 21, 2011.

This article has been peer reviewed. 
likelihood ratios of 0.2 (95\% CI $0.16-0.25)$ and 0.23 (95\% CI 0.18-0.28). ${ }^{12,13}$ In contrast, an American study looking at the efficacy of surgeon-directed EDUS in conjunction with physical examination as a predictor of IAI reported a sensitivity and a specificity of $100 \% .^{14}$

Bedside EDUS has recently become a mandatory component of adult emergency medicine residency training programs in both the United States and Canada. ${ }^{15-17}$ Recent American studies showed that EDUS is used by 74 to $96 \%$ of emergency physicians in adult settings compared to only 14 to $39 \%$ in pediatric centres. ${ }^{18-20}$

No data exist on the current use and perceived utility of bedside EDUS for pediatric trauma in Canada. The objectives of our study were to quantify the current availability and use of bedside EDUS for blunt trauma in Canadian pediatric centres and to identify any perceived barriers to the use of bedside EDUS in such centres.

\section{METHODS}

A 20-item, multiple-choice questionnaire was developed and administered using the Dillman Total Design Method for improving response rates. ${ }^{21}$ Using this method, the developed questionnaire employed large-point font and directed questions for ease of reading and completion. Important questions were asked first to increase respondent interest and survey completion. The survey was independently reviewed for content validity by four experts in the EDUS field. A pilot study was conducted by 20 physicians from four centres not included in the final distribution. Survey responses were tabulated to determine the distribution of responses, items generating high nonresponse rates, and the accuracy of responses.

Of the 20 questions in the survey instrument, 7 addressed basic demographics regarding the responding physician and site. One question addressed whether the responding site used EDUS to evaluate BAT. Physicians from sites currently using EDUS in the evaluation of BAT were asked to explain their perception of the utility of EDUS, the qualifications of those performing EDUS in their ED, the length of time since EDUS had been adopted by their ED, and the respondent's frequency of EDUS use. Those from sites not using EDUS in the evaluation of BAT were asked to describe reasons for not using EDUS to evaluate BAT, the respondent's level of interest in adopting EDUS into his or her current practice, and any clinical indications for which the respondent would like to use EDUS. The final two questions were for general comments and respondent site to calculate the response rate.

The study was facilitated by the Pediatric Emergency Research Canada (PERC) network, an organization comprising both pediatric emergency medicine (PEM) health care professionals and researchers that facilitate multicentre research. The PERC database was used to identify all Canadian pediatric EDs. The survey was then sent by e-mail to all physicians, working either full-time or part-time, who regularly work high-acuity shifts in an effort to exclude staff who were unexposed to pediatric trauma. High-acuity shifts were defined as any shift where patients are received by ambulance. The compiled list of physicians was developed from members of the Canadian Association of Emergency Physicians (CAEP), members of PERC, and staff lists provided from site directors for each pediatric centre (the latter of which proved to be most complete and was used for the survey distribution). The questionnaire was distributed between November 2009 and February 2010 to 12 pediatric EDs across Canada. Applying the Dillman approach to survey administration, ${ }^{21}$ the survey was distributed in four spaced mailings. The original e-mail contained the questionnaire and cover letter. A reminder e-mail was sent 1 week after the original e-mail, and replacement questionnaires were sent 4,6 , and 8 weeks after the original e-mail to nonrespondents. The questionnaire was sent to 12 site directors and 162 PEM physicians.

The questionnaire was distributed via SurveyMonkey, which tracked response rates from each individual pediatric centre while maintaining complete respondent anonymity. The study was approved by the McGill University Faculty of Medicine Institutional Review Board. Data are reported using descriptive statistics.

\section{RESULTS}

Of the 12 Canadian pediatric centres surveyed, 11 centres responded, for a centre response rate of $92 \%$. Individual response rates within each centre varied from 50 to $93 \%$, with three site response rates less than $70 \%$. From the responding sites, $100 \%$ of site directors responded to the questionnaire. The overall response 
rate from the 162 surveys sent to individual physicians was $65 \%$ (106 of 162), with an overall survey completion rate of $94 \%$.

The composition of responding physicians was $81 \%$ general pediatrics, $17 \%$ pediatric emergency medicine trained (Peds-EM), 9\% 5-year emergency medicine (EM-5 year), 5\% family medicine with 1 year emergency medicine, and 4\% family physicians.

Forty-five percent (5 of 11) of responding sites had ultrasonography currently available in the ED. Of these, $41 \%$ (32 of 77) of the responding physicians with ultrasonography available in their ED used bedside EDUS for the examination of BAT. Of those using bedside EDUS, the majority reported using it never or rarely (Figure 1). Although the reported frequency of use of bedside EDUS was low, its perceived utility was high, with $68 \%$ of users stating that ultrasonography positively affects patient care and management.

The presence of ultrasound machines in pediatric EDs is a new phenomenon. No Canadian EDs reported having ultrasonography capabilities for more than 5 years (Figure 2).

Bedside ultrasound examinations were reported to be performed by ED staff in $96 \%$ of cases. The remaining cases were reported to be performed by the on-call radiology staff and trauma surgeons. As the majority of staff working in pediatric settings are trained in pediatrics, in $34 \%$ of cases, pediatricstrained ED staff perform the ultrasound examination, EM-5 year staff complete $22 \%$ of examinations, family physicians perform 22\%, and emergency medicine residents complete $18 \%$ of examinations.

No apparent relationship existed between the volume of trauma patients seen at a particular facility and the presence or absence of a dedicated ED ultrasound machine, nor was there any apparent relationship between the number of years ED staff have been practicing and their use of ultrasonography.
We did find that EM-5 year staff working in both adult-only and pediatric-only sites were more likely to use their ultrasound training in the pediatric setting if ultrasonography were available to them. Seventy-five percent of 5 -year trained staff used ultrasonography in the pediatric context, $68 \%$ of them use it often, and $33 \%$ use it in all cases. Overall, $68 \%$ of them felt that it positively affected patient management. However, this population represented only $12 \%$ of those surveyed.

Figure 3 shows the reported impediments to the use of EDUS, both for the entire population and for the PEM-trained subgroup. Although PEM-trained physicians responses were generally similar to those of all respondents, they did differ regarding the perceived barriers to the use of bedside EDUS. A lack of training was perceived to be the most significant impediment by the entire study population; however, among the PEM-trained subgroup, a lack of equipment and training was reported to be most significant.

Of those not currently using bedside EDUS, 89\% were interested in its use for the evaluation of BAT and $81 \%$ believed that its implementation would have a positive impact on the evaluation and management of trauma patients. Many of those not interested in using ultrasonography for the evaluation of pediatric BAT still expressed an interest in incorporating ultrasonography in the evaluation of fracture reduction, wound infection assessment, intravenous access, and foreign body assessment.

\section{DISCUSSION}

The use of EDUS in the evaluation of BAT in adults is a common and widely accepted practice. ${ }^{8-10}$ Beyond the $\mathrm{ED}$, ultrasonography for trauma assessment has been adopted by military personnel for field use ${ }^{22}$ and as a screening test in mass-casualty situations. ${ }^{23,24}$ Although bedside ultrasonography has become a modality taught

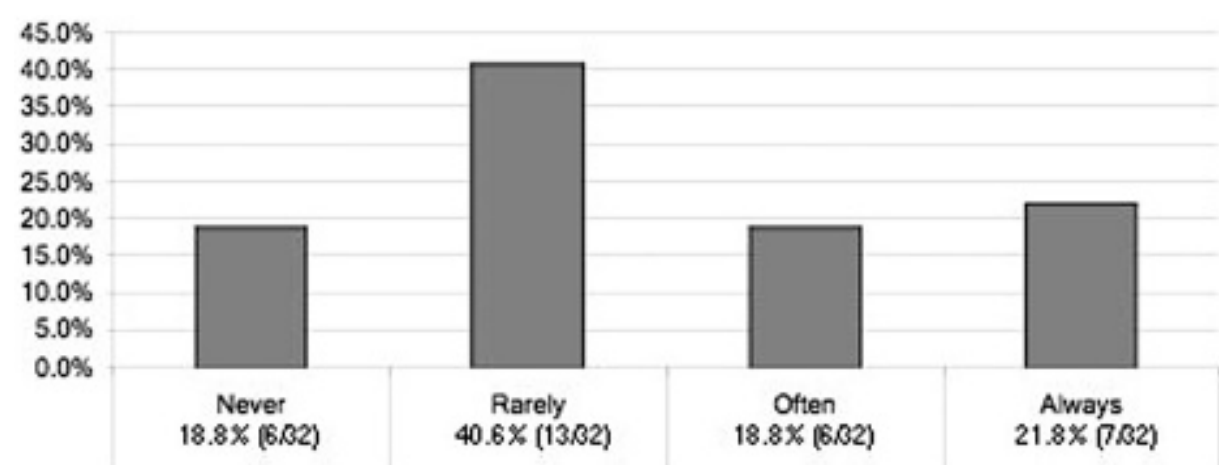

Figure 1. Frequency of use of bedside emergency department ultrasonography in the evaluation of blunt abdominal trauma. 


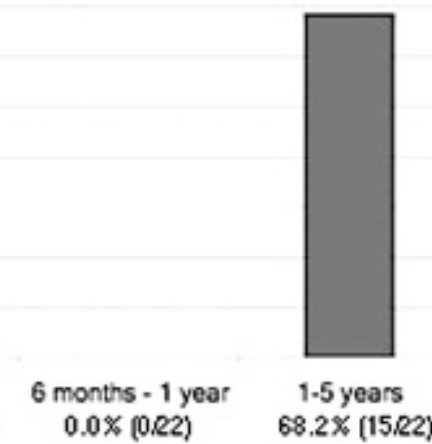

Figure 2. Duration of time ultrasonography has been present in emergency departments.

in both adult surgical and emergency medicine residency programs, ${ }^{15,16}$ this has not translated into the pediatric training environment. This may be due to the paucity of published evidence on the utility of EDUS in the pediatric setting. Although adult data cite sensitivities greater than $80 \%,{ }^{25}$ a recent meta-analysis reported the overall sensitivity in children to be as low as $66 \% .^{12}$

Our results indicate that the availability of ultrasonography in pediatric EDs remains relatively low at $45 \%$ compared to $89 \%$ of adult centres reported in an American study. ${ }^{20}$ Although this discrepancy has been described elsewhere, ${ }^{18-20}$ to our knowledge, our study is the first to explore the reasons for it. The main criticism of the use of EDUS in children has been its reported limited sensitivity. The explanation for these false negative results is that $40 \%$ of IAI in children is not associated with free fluid or is associated with amounts too small to be detected by ultrasonography. ${ }^{12,26}$ As nonoperative management of solid organ injuries has become common in pediatrics, it is arguable that many of these "missed injuries" or "false negatives" represent inconsequential injuries that would be managed by a period of observation. A study in 2003 reported that only $2.7 \%$ of false-negative ultrasound scans went on to require surgical management, whereas the rest were managed expectantly. ${ }^{27}$ More recent studies with newer generation ultrasound machines have found an 8 to $10 \%$ false negative rate; however, all of these cases were managed nonoperatively with observation. ${ }^{28,29}$ Although abdominal CT scans and serial physical examinations with an observation period have become the standard of care at most pediatric trauma centres, ${ }^{30}$ a study in 2004 found that only $5 \%$ of positive CT scans required surgical intervention. ${ }^{2}$ With this low intervention rate and the increased lifetime risk of fatal cancers associated with radiation from CT scans in childhood, ${ }^{4-7}$ the combination of serial EDUS and physical examinations may be both a safer and a more effective modality. ${ }^{14}$

Future research is needed to determine if there is an age group at which EDUS test performance drops off

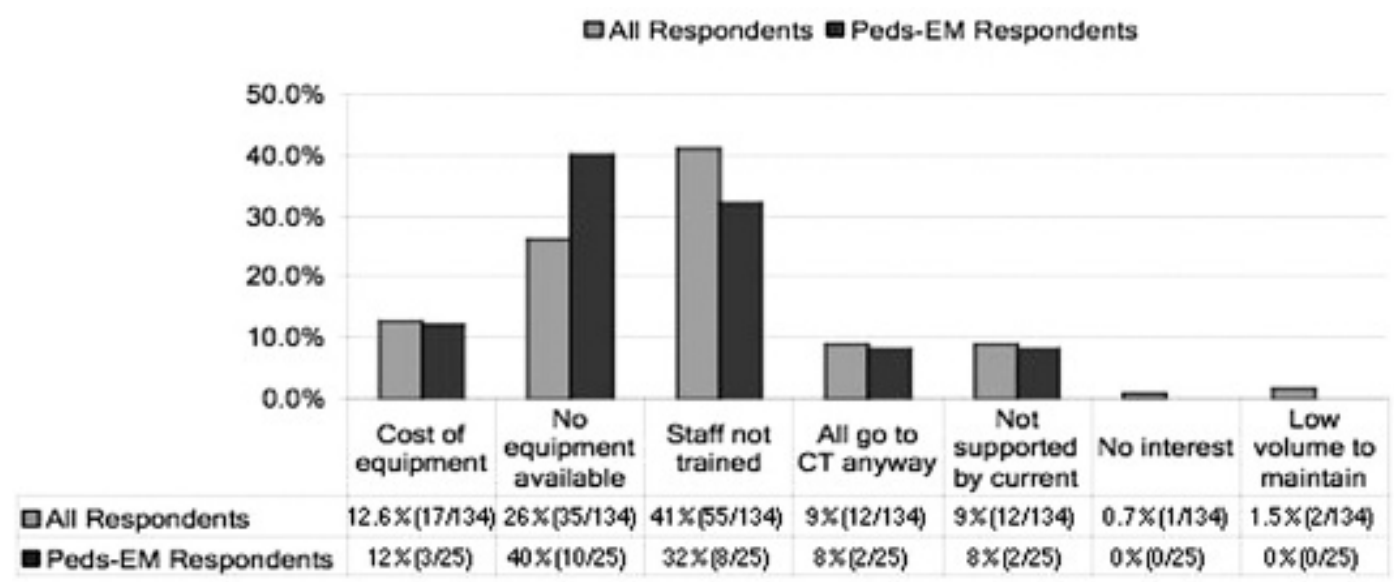

Figure 3. Main limitations to the use of bedside ultrasonography. CT = computed tomography; Peds-EM = pediatric emergency medicine trained. 
and whether EDUS can accurately detect all IAIs requiring surgical intervention, even if it fails to pick up cases that would be managed expectantly. Ultimately, a study to determine whether a difference in outcome exists between patients managed with current standard care (abdominal CT scan with serial physical examinations) and those managed with serial ultrasound and physical examinations in conjunction with an observation period is needed. If proven safe and effective, such an approach could limit the number of children being exposed to radiation and developing lethal cancers as a result.

Interestingly, and to our surprise, a lack of literature support was the reported reason for not incorporating ultrasonography into current pediatric trauma evaluation in only $9 \%$ of cases. Rather, physicians felt that a lack of adequate training in EDUS examination skills and a lack of equipment represented the main barriers to the use of ultrasonography in pediatrics $(41 \%$ and $26 \%$ of cases, respectively). Incorporating ultrasound training into PEM residency programs might overcome this impediment and increase EDUS use in this population.

Our study indicates that although the frequency of use of ultrasonography in the evaluation of BAT is relatively low at $41 \%$, the perceived utility remains high, with $68 \%$ of physicians using ultrasonography believing that it positively affects patient care, evaluation, and management. Of the five study sites with ultrasound capabilities, none had this modality available for more than 5 years, further illustrating the early stages of this technology in the pediatric setting. At the remaining six sites, $88 \%$ of physicians reported being interested in incorporating ultrasonography in the evaluation of BAT and $81 \%$ believed that it will have a positive impact on patient management. Our study also demonstrates that EM-5 year physicians working regularly in adult facilities are more likely to use ultrasonography in the pediatric setting, a finding that is likely due to the adoption of ultrasound training within adult EM residency programs and a familiarity with the equipment. This provides further evidence that including EDUS training in pediatric residency programs might narrow the gap between the use of ultrasonography in pediatric and adult settings.

The primary limitation of our study is that the findings were derived from a survey, and the results depict the current reported use and perceived utility of ultrasonography at a static point in time. However, given our relatively high response rate of $65 \%$ and high survey completion rate of $94 \%$, we believe that our results are an accurate reflection of the beliefs and practices of pediatric emergency physicians. Although our results suggest that including ultrasound training in pediatric emergency residency programs might increase the use of ultrasonography in the evaluation of pediatric traumas, we did not propose a method for incorporating ultrasound training in pediatric programs. Further research is necessary to both design and determine the effect of ultrasound training programs. A final limitation was the lack of information from one pediatric centre, the only site that did not respond to the questionnaire. However, the ED director at this site indicated that their site does not currently have a dedicated ultrasound machine and does not use EDUS to evaluate BAT because they see a very limited number of severe trauma cases per year, and most investigations and imaging are done by trauma and radiology services. If these data had been included, the availability of EDUS in the pediatric setting we reported would have been even lower at 42\% (5 of 12).

\section{CONCLUSIONS}

Bedside EDUS is currently used in almost half of pediatric trauma centres, a frequency that is significantly lower than that of adult centres. Physicians in pediatric centres who use ultrasonography report that it has a high utility, and the great majority of physicians at pediatric centres without ED ultrasonography plan to incorporate it in the future. The main reported barriers to its use are a lack of training and a lack of equipment availability.

Competing interests: None declared.

\section{REFERENCES}

1. Centers for Disease Control and Prevention. Deaths, leading causes for 2004. Natl Vital Stat Rep 2004.

2. Fenton SJ, Hansen KW, Meyes RL, et al. CT scan and the pediatric trauma patient-are we overdoing it? 7 Pediatr Surg 2004;39:1877-81, doi:10.1016/j.jpedsurg.2004.08.007.

3. Taylor GA, Eichelberger MR. Abdominal CT in children with neurologic impairment following blunt trauma. Ann Surg 1989;210:229-33, doi:10.1097/00000658-19890800000016.

4. Brenner D, Elliston C, Hall E, et al. Estimated risks of radiation-induced fatal cancer from pediatric CT. $A 7 R A m \mathcal{F}$ Roentgenol 2001;176:289-96. 
5. Slovis TL. Children, computed tomography radiation dose, and the As Low As Reasonably Achievable (ALARA) concept. Pediatrics 2003;112:971-2, doi:10.1542/peds. 112.4.971.

6. Brenner DJ. Estimating cancer risks from pediatric CT: going from the qualitative to the quantitative. Pediatr Radiol 2002;32:228-31, doi:10.1007/s00247-002-0671-1.

7. Jindal A, Velmahos GC, Rofougaran R. Computed tomography for evaluation of mild to moderate pediatric trauma: are we overusing it? World 7 Surg 2002;26:13-6, doi:10.1007/ s00268-001-0174-5.

8. Boulanger BR, McLellan BA, Brenneman FD, et al. Emergent abdominal sonography as a screening test in a new diagnostic algorithm for blunt trauma. 7 Trauma 1996; 40:867-74, doi:10.1097/00005373-199606000-00003.

9. McKenney MG, Martin L, Lentz K, et al. 1,000 consecutive ultrasounds for blunt abdominal trauma. 7 Trauma $1996 ; 40$ : 607-10, doi:10.1097/00005373-199604000-00015.

10. Rozycki GS, Ochsner MG, Jaffin JH, et al. Prospective evaluation of surgeons' use of ultrasound in the evaluation of trauma patients. 7 Trauma 1993;34:516-26, doi:10.1097/ 00005373-199304000-00008.

11. Melniker LA, Leibner E, McKenney MG, et al. Randomized controlled clinical trial of point-of-care, limited ultrasonography for trauma in the emergency department: the first sonography outcomes assessment program trial. Ann Emerg Med 2006;48:227-35, doi:10.1016/j.annemergmed.2006. 01.008 .

12. Holmes JF, Gladman A, Chang CH. Performance of abdominal ultrasonography in pediatric blunt trauma patients: a meta-analysis. $\mathcal{f}$ Pediatr Surg 2007;42:1588-94, doi:10.1016/j.jpedsurg.2007.04.023.

13. Stengel D, Bauwens K, Sehouli J, et al. Systematic review and meta-analysis of emergency ultrasonography for blunt abdominal trauma. Br 7 Surg 2001;88:901-12, doi:10.1046/ j.0007-1323.2001.01777.x.

14. Suthers SE, Albrecht R, Foley D, et al. Surgeon-directed ultrasound for trauma is a predictor of intra-abdominal injury in children. Am Surg 2004;70:164-7.

15. Hockberger RS, Binder LS, Graber MA, et al. The model of the clinical practice of emergency medicine. Ann Emerg Med 2001;37:745-70, doi:10.1067/mem.2001.115495.

16. Heller MB, Mandavia D, Tayal VS, et al. Residency training in emergency ultrasound: fulfilling the mandate. Acad Emerg Med 2002;9:835-9, doi:10.1111/j.1553-2712.2002.tb02174.x.

17. Royal College of Physicians and Surgeons of Canada. Objectives of training in emergency medicine. Policies and procedures booklet. 2008.
18. Baka AG, Delgado CA, Simon HK. Current use and perceived utility of ultrasound for evaluation of pediatric compared with adult trauma patients. Pediatr Emerg Care 2002;18:163-7, doi:10.1097/00006565-200206000-00003.

19. Chen L, Santucci A. Probing the availability of emergency ultrasound in pediatrics [abstract]. Pediatr Emerg Care 2006; 22:688, doi:10.1097/00006565-200609000-00041.

20. Scaife E, Fenton SJ, Hansen KW, et al. Use of focused abdominal sonograpy for trauma at pediatric and adult trauma centers: a survey. 7 Pediatr Surg 2009;44:1746-9, doi:10.1016/j.jpedsurg.2009.01.018.

21. Dillman DA. Mail and telephone surveys: the Total Design Method. New York: Wiley; 1978.

22. Brooks AJ, Price V, Simms M. FAST on operational military deployment. Emerg Med 7 2005;22:263-5, doi:10.1136/ emj.2004.014308.

23. Sarkisian AE, Khondkarian RA, Amirbekian NM, et al. Sonographic screening of mass casualties for abdominal and renal injuries following the 1988 Armenian earthquake. 7 Trauma 1991;31:247-50.

24. Fernandez L, McKenney MG, McKenney KL, et al. Ultrasound in blunt abdominal trauma. 7 Trauma 1998;45: 841-8, doi:10.1097/00005373-199810000-00047.

25. Dolich MO, McKenney MG, Varela JE, et al. 2,576 ultrasounds for blunt abdominal trauma. 7 Trauma 2001; 50:108-12, doi:10.1097/00005373-200101000-00019.

26. Taylor GA, Sivit CJ. Posttraumatic peritoneal fluid: is it a reliable indicator of intraabdominal injury in children. 7 Pediatr Surg 1995;30:1644-8, doi:10.1016/0022-3468 (95)90442-5.

27. Akgür FM, Tanyel FC, Akhan O, et al. The place of ultrasonographic examination in the initial evaluation of children sustaining blunt abdominal trauma. 7 Pediatr Surg 1993;28:78-81, doi:10.1016/S0022-3468(05)80361-6.

28. Coley BD, Mutabagani KH, Martin LC, et al. Focused abdominal sonography for trauma (FAST) in children with blunt abdominal trauma. 7 Trauma 2000;48:902-6, doi:10.1097/00005373-200005000-00014.

29. Soundappan SV, Holland AJ, Cass DT, et al. Diagnostic accuracy of surgeon-performed focused abdominal sonography (FAST) in blunt paediatric trauma. Injury 2005;36:9705, doi:10.1016/j.injury.2005.02.026.

30. Taylor GA, Fallat ME, Potter BM, et al. The role of computed tomography in blunt abdominal trauma in children. 7 Trauma 1988;28:1660-4, doi:10.1097/00005373198812000-00008. 